\title{
ORIENTATION CORRELATION IN 72.5\% STRAINED OFE COPPER
}

\author{
T. A. MASON and B. L. ADAMS \\ Department of Mechanical Engineering, Yale University, New Haven, CT
}

\begin{abstract}
Misorientation Correlation Functions, MCFs, for a 72.5 percent cold-rolled OFE copper plate were calculated for groups of local lattice orientation measurements taken from the rolling-normal and rolling-transverse planes of the sample. The MCFs' dependence upon sample direction, orientation pair selection and the spatial separation of orientation measurements was explored. A preferred misorientation region in Euler space was identified and found to contain misorientations corresponding to coincides of $\Sigma 3, \Sigma 33 \mathrm{c}, \Sigma 37 \mathrm{c}$ and $\Sigma 43 \mathrm{c}$. MCFs associated with orientations of interest were found to exhibit unique characteristics.
\end{abstract}

KEY WORDS Local texture measurements, Textural orientation correlation, Preferred misorientation

\section{INTRODUCTION}

Recent work by Haessner, Pospiech and Sztwiertnia (1983), Adams et al. (1987), Wang, Adams and Morris (1990) and Sztwiertnia and Haessner (1992) provided evidence that the description of polycrystals as aggregates of grains which are spatially uncorrelated with respect to orientation is not complete and that an orientation coherence or correlation mesostructure exists beyond the scale of the grain size. This means that there may be a correlation between the orientation of neighboring grains depending on the material and its processing history. This study was aimed at exploring the intercrystalline orientation correlation, or preferred misorientation, due to large strain deformation of copper and characterizing that correlation in misorientation space.

Lattice orientation was determined for groups of points on square grids which were randomly placed on the rolling-normal, RN, and rolling-transverse, RT, planes. The texture or the Orientation Distribution Function, ODF, of the material was estimated from the individual orientation measurements. Conditional Misorientation Correlation Functions, MCFs, were generated by three schemes of sorting the pairs of orientation measurements. These were: (1) collecting all spatial first-neighbors regardless of sample direction, (2) sorting first through fourth-neighbors with distinct sample directions noted, and (3) sorting with one orientation of the pair chosen by its proximity to an orientation of special interest in Euler space. These conditional MCFs correspond directly to Sztwiertnia and Haessner's "partial" MODFs with weight factors of one for the above noted misorientations and zero otherwise.

The misorientation correlation functions will be represented by contour plots of isolines in an asymmetric region of Euler space. This reduced volume of Euler 
space presents the minimum asymmetric portion of the MCFs as well as its boundary containing misorientations corresponding to all CSL boundaries for $\Sigma \leq 49$.

The Textural Orientation Correlation, TOC, was used as a measure of the statistical significance of MCF features and their dependence on the sample texture alone. Rescaled misorientation distributions were calculated by subtracting the TOC from the calculated MCFs. The resulting functions reveal the strength of MCF features with respect to those of the TOC.

\section{MATERIAL AND MEASUREMENTS}

The material studied was oxygen-free electrical grade (OFE) copper plate which was unidirectionally cold-rolled to a 72.5 percent reduction. Initial examination of samples in the scanning-electron-microscope (SEM) yielded poor quality electron backscatter diffraction pattern (EBSP) images. The EBSP images were required to manually determine lattice orientations and poor contrast or total lack of discernible bands in the images precluded this determination. In an attempt to improve the clarity of these patterns, the samples were recovery annealed at $100^{\circ} \mathrm{C}$ for a period of one-half hour. The resulting patterns could then be analyzed after the images were averaged and enhanced by background subtraction.

Two samples were prepared from the center of the recovery annealed specimens. The surface of interest of the first sample contained the rolling and normal directions (RN) and the second contained the rolling and transverse (RT) directions. These surfaces were then mechanically polished and subsequently electropolished for examination in the SEM. Due to the highly-strained state of the copper, it was not possible to etch the surfaces to highlight the boundaries of the grains. Any attempt to etch the surface resulted in a rough surface which could not be analyzed using EBSP methods in the SEM. The surfaces were scanned in the SEM to estimate the average diameter of sub-grains by noting changes in the EBSP images. The average diameter was estimated at $2.5 \mu \mathrm{m}$. Determination of lattice orientation was then performed on square grids located randomly on the two surfaces with the grid axes aligned with the sample directions. Each grid or set of measurements consisted of twenty-five orientations $(5 \times 5)$ spaced $2.5 \mu \mathrm{m}$ apart.

The EBSP patterns were much improved following the recovery anneal; but, as mentioned above, image processing was still required before they could be analyzed by hand with any confidence. The total number of usable orientations obtained was 1143 and 1149 orientations for the RN and RT planes, respectively.

\section{FUNCTION DEFINITIONS}

All distribution and correlation functions discussed in this study were generated from discrete orientation or misorientation matrices using generalized spherical harmonic expansions as described by Bunge (1982). The orientations were approximated as Gaussian functions in Euler space with five degree half-widths. 
The coefficients describing each data set were obtained with the harmonic equations expanded to 22 nd order. The ODF function values are determined on a three-dimensional grid in Euler space with five degree spacing in each of the angles $\left(\varphi_{1}, \phi, \varphi_{2}\right)$. The MCF function values are calculated on a grid with differential spacing to accommodate use of the asymmetric domain of cubic-cubic misorientations discussed below. The spacing for $\varphi_{1}$ and $\varphi_{2}$ will be five degrees while the spacing for $\phi$ will be two degrees. Any spatial or directional functionality of these functions is found in the data selection process and not in the harmonic expansions.

The MCFs are probably density functions, $m(\Delta g)$, which describe the probability of obtaining a pair of orientations related by a given misorientation, $\Delta g$. This is expressed in Eq. (1) where $d N / N$ is the number fraction of measurement pairs exhibiting misorientations in a neighborhood of measure $d \Delta g$ about $\Delta g$ in cubic-cubic misorientation space $E_{c}$.

$$
\frac{d N}{N}=m(\Delta g) d \Delta g
$$

The MCF is normalized according to

$$
\int_{E_{c}} m(\Delta g) d \Delta g=1 .
$$

Here the MCF is distinguished from the Misorientation Distribution Function or MODF because the misorientations used in this study do not necessarily span a grain boundary. The experimental challenges mentioned in the previous section forced this study to depend upon the misorientation statistics of the grids instead of explicit orientation pairs at distinct boundaries.

As noted above, three types of conditional MCFs were calculated. These conditional functions are defined in Eq. (3).

$$
\frac{d N}{N} \mid \ldots=m(\Delta g \mid \ldots) d \Delta g
$$

The vertical line after $\Delta g$ indicates that what follows are the sorting conditions applied to the calculations of $\Delta g$. The conditional MCF's were normalized in a manner analogous to Eq. (2) viz

$$
\int_{E_{c}} m(\Delta g \mid \ldots) d \Delta g=1 .
$$

The first conditional MCFs were generated from all first-neighbor misorientations in the RN and RT planes regardless of sample direction. These can be expressed as

$$
m\left(\Delta g \mid r \in\left\{r_{1,0}, r_{0,1}\right\}\right)
$$

where $r$ is defined from Figure 1 as

$$
r_{i, j}=X_{\alpha+i, \beta+j}-X_{\alpha, \beta}
$$

Here $\alpha$ and $\beta$ assume values ranging from one to five. The condition applied in Eq. (5) is that the orientation pairs used to calculate misorientations are first-neighbor pairs in either of the sample directions available in a given grid. 


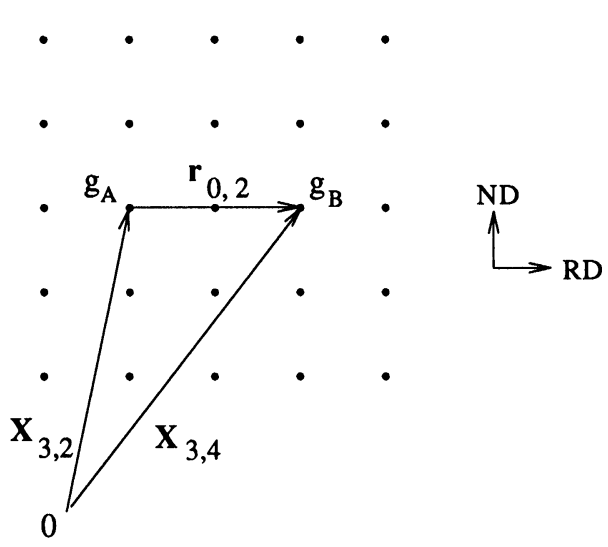

Figure 1 Definition of $r$-vectors used in conditional misorientation correlation functions.

The second group of conditional MCFs was generated from data sets which discriminated between sample direction and also orientation pair separation. Thus eight distributions were found by selecting the rolling and normal directions and the four available separation distances in the RN data grids. Typical distributions were

$$
m\left(\Delta g \mid r \in\left\{r_{1,0}\right\}\right), m\left(\Delta g \mid r \in\left\{r_{0,3}\right\}\right), \text { etc. }
$$

It should be noted that only orientation pairs in the rolling and normal directions were used in this work. No diagonal pairs were considered.

The third set of conditional MCFs was obtained by considering spheres centered at designated orientations of interest in Euler space, noting all orientations which resided in those spheres. The criterion used to determine residence in a sphere was whether the minimum rotation angle required to achieve coincidence with the orientation of interest was less than ten degrees. The misorientations between these noted orientations and their spatial first-neighbors were then calculated. These distributions can be expressed as

$$
m\left(\Delta g \mid r \in\left\{r_{1,0}, r_{0,1}\right\}, \cos ^{-1}\left(\frac{1-\operatorname{trace}\left[g g_{A}^{T}\right]}{2}\right)<10^{\circ}\right)
$$

where $g_{A}$ denotes the first orientation in a spatial orientation pair and $g$ denotes the designated orientation of interest.

It is beneficial to provide some means of comparing MCFs to a normal or random misorientation distribution. Several methods have been used in attempts to normalize the MCF; however, due to numerical instabilities inherent in taking the quotient of functions, this study subtracted a reference function, the Textural Orientation Correlation or TOC, (see Bunge (1982), Plege (1987) and Zhao, Adams and Morris (1988)), in order to make comparisons. The TOC describes what the texture alone would predict for the misorientation distribution. Thus differences between the TOC and an experimental MCF reveal those features of the MCF which exhibit statistically significant orientation correlation. The TOC is 
defined as

$$
m^{0}(\Delta g)=\int_{E} f(g) f(\Delta g \cdot g) d g,
$$

where $g$ denotes orientation, $\Delta g$ misorientation and $f(g)$ the ODF. The integration is over $E$, the space of physically distinct orientations of a cube. In terms of a harmonic expansion this definition is equivalent to

$$
m^{0}(\Delta g)=\sum_{\lambda, v} \sum_{\mu, \mu^{\prime}} \frac{C_{\lambda}^{\mu \nu} C_{\lambda}^{\mu^{\prime} v}}{2 \lambda+1} \ddot{T}_{\lambda}^{\mu^{\prime} \mu}(\Delta g)
$$

where the coefficients $C_{\lambda}^{\mu \nu}$ are those calculated for the ODF, and $\ddot{T}_{\lambda}^{\mu^{\prime} \mu}(\Delta g)$ are the generalized spherical harmonics with cubic-cubic symmetry. It has been noted by Sztwiertnia and Haessner (1992) that when conditional or partial distributions are under examination that another possible rescaling scheme would be to use the first-neighbor MCF as the reference in place of the TOC. The choice of the scaling quantity can be made in accordance with the desired analysis.

\section{RESULTS AND DISCUSSION}

The specimens exhibited a relatively well defined rolling texture as evidenced by the Orientation Distribution Function or ODF. The ODF was first calculated for 1143 orientations obtained from the RN plane assuming cubic crystal and triclinic sample symmetries. Comparison of this ODF with that calculated using the same orientation matrices and assuming cubic-orthotropic symmetry showed that the data reflected a well developed orthotropic symmetry. The cubic-orthotropic ODF is shown in Figure 2. The maximum peak height of this function was found to be 10.4 times random. It is noted that the ODF exhibited minor discontinuities in the $\beta$-fiber which are not characteristic of a general rolling texture; these were credited to the function having been generated from finite numbers of single orientation measurements. Discontinuity aside, the ODF agrees favorably with the rolling textures given for copper of comparable deformation by Bunge (1982) and Hirsch and Lücke (1988).

The TOC corresponding to the ODF in Figure 2 is shown in Figure 3. The boundary of the asymmetric domain of intercrystalline misorientations in cubic materials, as proposed by Zhao and Adams (1987), is given by the heavy line. The significance of the asymmetric region is seen when it is realized that all Coincidence-Site Lattice or CSL boundaries with $\Sigma \leq 49$ lie on the boundary of this region. Most of the misorientations residing on the boundary generally possess group multiplicity greater than one while those residing inside the region have multiplicity of only one. Another important feature of this region is that rotations about the $\langle 111\rangle$ axis fall at the two receding corners, marked as $\mathrm{A}$, while rotations about $\langle 110\rangle$ appear at the rounded corner receding from the bottom-right of the $\phi$ sections, marked B. Rotations about $\langle 112\rangle$ and $\langle 210\rangle$ occur at locations along the left or bottom boundary while rotations about $\langle 332\rangle$ and $\langle 221\rangle$ occur along the right or top boundary. It should be noted that unlike the corners of the region, the $\left(\varphi_{1}, \phi, \varphi_{2}\right)=\left(45^{\circ}, \phi, 45^{\circ}\right)$ location in the center of 

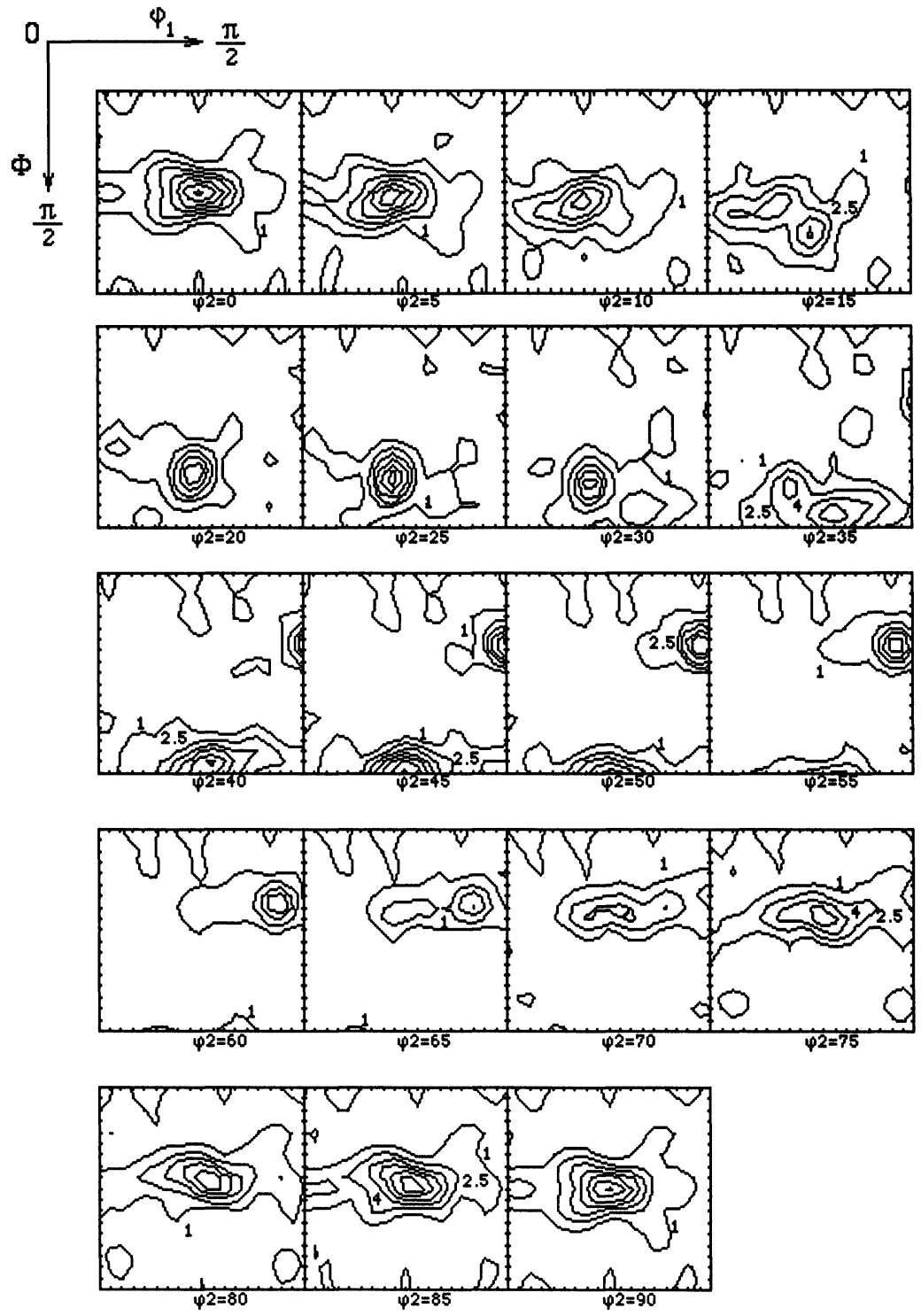

Figure 2 Orientation Distribution Function for $\mathrm{Rn}$ plane in $72.5 \%$ strained $\mathrm{Cu}$. Contour levels of 1 , $2.5,4,5.5,7,8.5$, and 10 times random.

each $\phi$ section does not correspond to rotations about a 'stationary' axis. This means that as $\phi$ descends from $90^{\circ}$ the axis of rotation at the center of the section changes.

Despite the initial awkardness of this asymmetric region, its use obviates the need to transform from Euler space, where computation is more convenient, to axis-angle space, where traditional representation is more intuitive. In addition, 

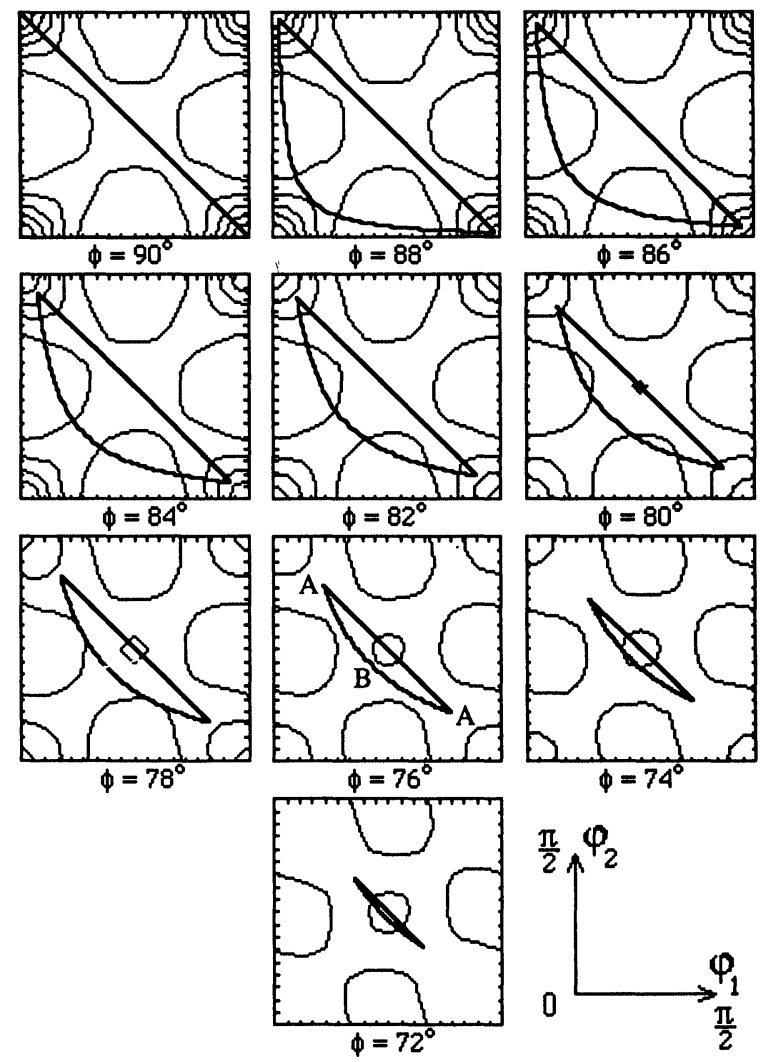

Figure 3 Textural Orientation Correlation from ODF in Figure 2. Contour levels of 0.51, 0.92, 1.32, $1.73,2.13,2.54$ and 2.94 .

the representation of misorientation in Euler space can be of great value when dealing with small angle misorientations. As the rotation angle approaches zero in axis-angle space, the axis of rotation becomes ambiguous.

The rescaled MCF, denoted as $c(\Delta g)$, was obtained from Eq. (11), where $m(\Delta g)$ is the experimental MCF.

$$
c(\Delta g)=m(\Delta g)-m^{0}(\Delta g)
$$

It should be noted that the integral of $c(\Delta g)$ over $E_{c}$ yields zero. When the TOC is subtracted from an MCF, the resulting rescaled MCF reveals the relative intensities of features in the MCF versus those in the TOC.

First-neighbor misorientations were calculated for all available orientation pairs in the RN and RT grids. MCFs corresponding to Eq. (5) were calculated enforcing cubic-cubic symmetry. The MCFs exhibited a very large peak at misorientations corresponding to small angles of rotation. The major contributor to these high densities was large numbers of measurement pairs which both fell in the same grain or sub-grain. Misorientations which had an absolute rotation angle of less than ten degrees were considered self-correlating. These small angle rotations dominate the function values of the MCFs to the extent that any other 


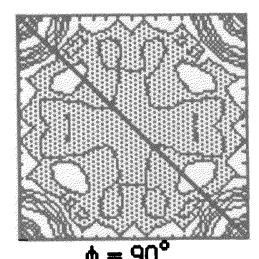

$\phi=90^{\circ}$
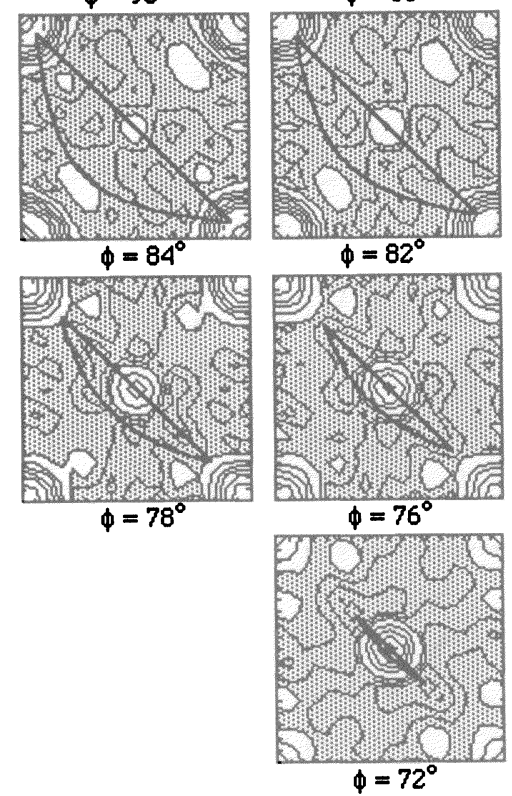
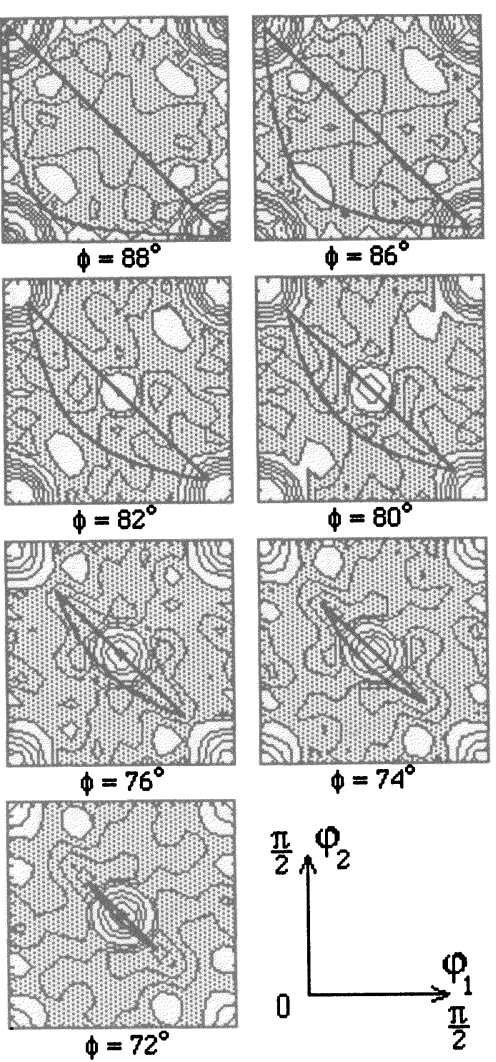

Figure 4 Rescaled Misorientation Correlation Function for first-neighbor misorientations with rotation angle greater than $10^{\circ}$. Shaded regions are negative. Contour levels of $-1.0,-0.75,-0.5$, $-0.25,0,0.73,1.47,2.2,2.93,3.67$ and 4.4 .

components of the misorientation distribution are obscured. If these small angle misorientations are not used in the MCF calculations, a second component of the misorientation texture becomes evident in both the RN and RT planes.

In Figure 4, the rescaled MCF for the RN plane displays these features. The remnant of the self-correlating component can be seen as a plateau at the corners of the constant $\phi$ sections with the second component emerging from the center of the sections as $\phi$ descends from 90 to $72^{\circ}$. The shaded portions correspond to regions in misorientation space where the MCF features were weaker than those predicted from the texture alone, giving rise to negative values. Conversely, the unshaded or positive regions reveal where the MCF exhibited features of greater strength than those of the TOC. Using misorientation angle as a metric, the distance from the theoretical $\Sigma 3$ misorientation to the zero contours of the second component in the rescaled RN MCF can be roughly described as an ellipsoid with its major axis along the $\phi$ axis and minor axes of equal length along the $\varphi_{1}$ and $\varphi_{2}$ axes. The difference between the major and minor axes was approximately 16 percent. Thus the misorientation can be viewed as a non-spherical spread about the $\Sigma 3$ type misorientation centered at $\left(\varphi_{1}, \phi, \varphi_{2}\right)=(45,70.53,45)$. This 
misorientation can be viewed as a rotation of $60^{\circ}$ about a $\langle 111\rangle$ axis. The ellipsoid contains misorientations whose axis-angle parameters correspond to four special CSL type boundaries. These are the $\Sigma 3, \Sigma 33 \mathrm{c}, \Sigma 37 \mathrm{c}$, and $\Sigma 43 \mathrm{c}$ (cf. Adams, Zhao and Grimmer, 1990). The rescaled MCF for the RT plane showed that the only major deviation from the TOC in that plane was the strong component at small angle misorientations.

The results of the second approach to generating conditional misorientation distributions, given by Eq. (7), showed that self-correlation occurs over distances exceeding four times the assumed grain diameter. It was also apparent that there is a difference in the orientation correlation between the rolling and normal sample directions. Comparison of the direction dependent MCFs revealed that the second component of the misorientation texture in the normal direction exhibited half the strength of that in the rolling direction for first-neighbors. By the fourth-neighbor separation, this second component had essentially disappeared in the normal direction while it still retained three-quarters of its first-neighbor strength in the rolling direction. The survival of the self-correlating component and not the second of the misorientation texture in the normal direction could possibly be credited to an effect of the original grain size.

The third scheme for calculating misorientations, given by Eq. (8), was used to calculate six MCFs. The first MCF was calculated using the six largest peaks (greater than 2.5 times random) of the ODF in Figure 2 as orientations of interest or sphere centers. Subsequently the five major theoretical texture components, C, $\mathrm{S}, \mathrm{B}, \mathrm{G}$ and $\mathrm{W}$, were used as sphere centers. A separate MCF was calculated for each of these data sets. The orientations used as sphere centers are listed in Table 1.

The resulting MCFs again exhibited a high frequency of small angle misorientations, which when ignored, generally yielded the two-component misorientation texture reported above. The MCF generated from the ODF peak orientations was essentially the same as that obtained using all first-neighbor misorientations. The MCFs derived from spheres centered on Copper and Brass orientations show only slight variation from the first-neighbor or ODF peak MCFs. These MCFs again exhibit the remnant of a strong self-correlating component and a second component centered on the $\Sigma 3$ misorientation.

The Cube MCF, shown in Figure 5, exhibited a strong component located approximately at the $\langle 111\rangle$ position in all of the $\phi$ sections for $\phi$ less than $86^{\circ}$. The $\langle 111\rangle$ rotation axes positions in the first three $\phi$ sections fall in a "well" left by the removal of all misorientations less than ten degrees. It is of interest that no peak develops at the $\Sigma 3$ position. The features in the $\phi=90^{\circ}$ section consist of

Table 1 Orientations used as sphere centers.

\begin{tabular}{llll}
\hline ODF Peak & $\left(\varphi_{1}, \Phi, \varphi_{2}\right)$ & Component & $\left(\varphi_{1}, \Phi, \varphi_{2}\right)$ \\
\hline 1 & $(40,75,25)$ & Cu (Copper) & $(90,35.3,45)$ \\
2 & $(44,45,90)$ & S & $(56.8,29.2,63.4)$ \\
3 & $(46,90,45)$ & B (Brass) & $(35.3,45,90)$ \\
4 & $(82,41,78)$ & G (Goss) & $(0,45,90)$ \\
5 & $(83,90,45)$ & W (cube) & $(0,0,0)$ \\
6 & $(90,32,45)$ & & \\
\hline
\end{tabular}



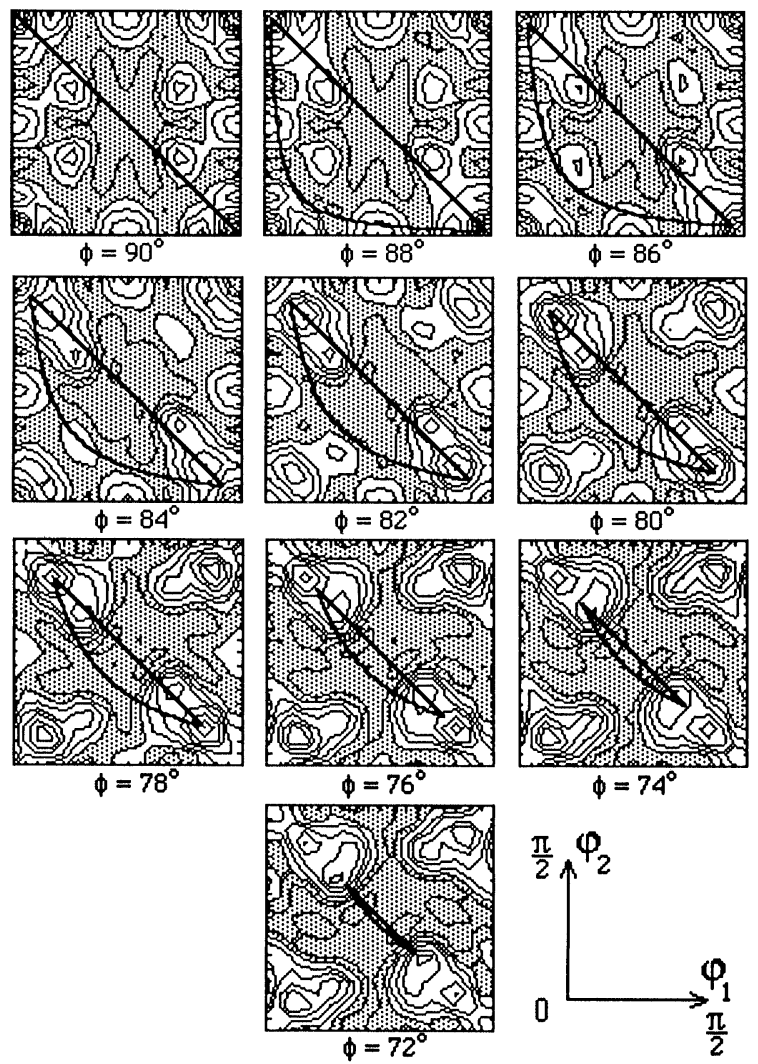

Figure 5 Rescaled Misorientation Correlation Function for first-neighbor misorientations associated with the Cube texture component. No misorientations less than $10^{\circ}$ are included. Contour levels of $-3.0,-2.25,-1.5,-0.75,0,0.75,1.5,2.25,3.0,3.75$ and 4.5 .

many symmetric pairs of peaks of height between 1.5 and 2.25 . These features merge to form a set of four almost symmetric peaks near the corners of the section. The pairs of peaks lying on the $\varphi_{1}+\varphi_{2}=90^{\circ}$ line exhibit a slight bifurcation or twin summits. These two summits attain their maximums at different values of $\phi$. The summit nearest the section corner peaks at a strength of 4.52 at $\left(15^{\circ}, 78^{\circ}, 75^{\circ}\right)$ whereas the inner summit attains a strength of 3.06 at $\left(25^{\circ}, 72^{\circ}, 60^{\circ}\right)$. The peak of 4.52 is the maximum for this MCF.

The Goss MCF, shown in Figure 6, exhibits a peak developing at $\left(45^{\circ}, \phi, 45^{\circ}\right)$ as $\phi$ descends from $90^{\circ}$ with the peak breaking into a pair of twin summits astride the $\varphi_{1}+\varphi_{2}=90^{\circ}$ line beginning in the $\phi=78^{\circ}$ section. The center peak attains a maximum of 1.56 at $\left(45^{\circ}, 82^{\circ}, 45^{\circ}\right)$ while the two peaks present on each side of the asymmetric region's boundary reach their peaks at values and positions of 1.64 and $\left(40^{\circ}, 76^{\circ}, 40^{\circ}\right)$ and 1.79 and $\left(50^{\circ}, 72^{\circ}, 25^{\circ}\right)$. In this case the maximum peak value, 3.17 , is found at $\left(0^{\circ}, 78^{\circ}, 90^{\circ}\right)$.

The MCF obtained from the $\mathrm{S}$ component exhibited no self-correlating component. The MCF for the S component is given in Figure 7. A set of three preferred misorientation peaks originally lie symmetrically above and below the 

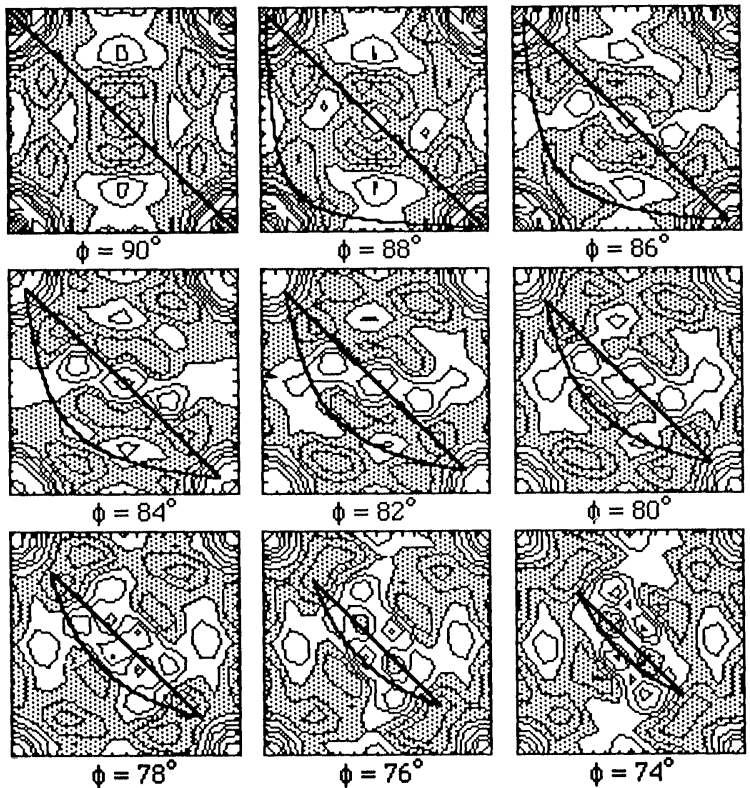
$\phi=80^{\circ}$
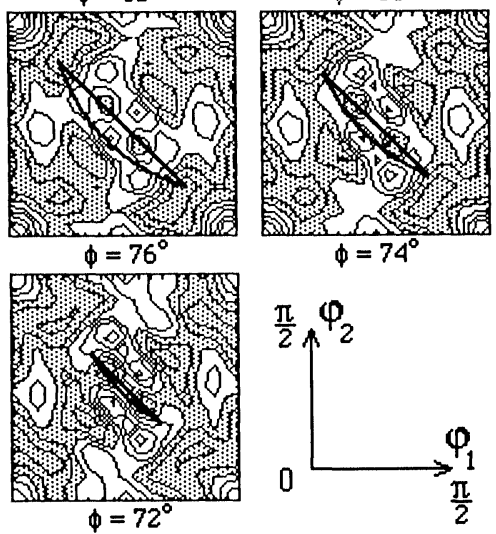

Figure 6 Rescaled Misorientation Correlation Function for first-neighbor misorientations associated with the Goss texture component. No misorientations less than $10^{\circ}$ are included. Contour levels of $-1.5,-1.0,-0.5,0,0.5,1.0,1.5,2.0,2.5$ and 3.0 .

center position but as $\phi$ descends from $90^{\circ}$ the regions shift to a pair of broad peaks on either side of a center peak and two sets of weaker features near the upper-left and lower-right corners of the $\phi$ sections. The middle peak at $\left(45^{\circ}, 90^{\circ}\right.$, $30^{\circ}$ ) had a maximum strength of 2.76 and the peak on the asymmetric boundary was 1.96 at $\left(20^{\circ}, 90^{\circ}, 70^{\circ}\right)$. The third peak reached its maximum of 2.52 at $\left(20^{\circ}\right.$, $86^{\circ}, 70^{\circ}$ ). These features changed to a center peak of 2.8 (the MCF maximum) at $\left(45^{\circ}, 72^{\circ}, 45^{\circ}\right)$ with symmetric peaks of 2.47 on either side at $\left(70^{\circ}, 72^{\circ}, 50^{\circ}\right)$ and $\left(20^{\circ}, 72^{\circ}, 40^{\circ}\right)$. The peaks near the corners have maximums of approximately 2 with positions symmetric in $\varphi_{1}$ and $\varphi_{2}$ to $\left(85^{\circ}, 72^{\circ}, 25^{\circ}\right)$.

Due to the relatively large spacing of the function values used in the comparisons above, all of the peak orientations for the Cube, Goss and S MCFs were recalculated on finer grids with one degree spacing in the neighborhood of the peaks. This provided more precision when transforming the Euler angles into axis-angle pairs. The axis-angle parameterizations corresponding to the peaks discussed above are listed in Table 2 . Two preferred orientation differences are especially worthy of note. They are the $40^{\circ}\langle 111\rangle$ rotation associated with the cube component and the $60^{\circ}\langle 111\rangle$ rotation associated with the $\mathrm{S}$ component. 


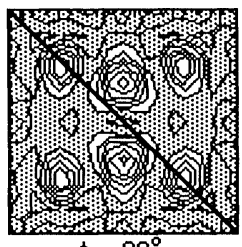

$\phi=90^{\circ}$

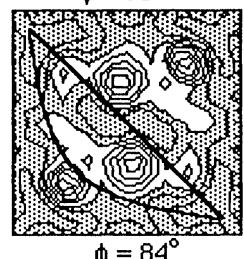

$\phi=84^{\circ}$

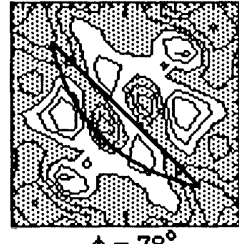

$\phi=78^{\circ}$

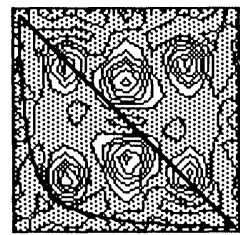

$\phi=88^{\circ}$
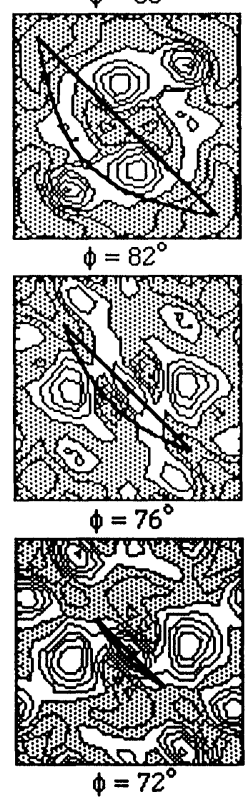

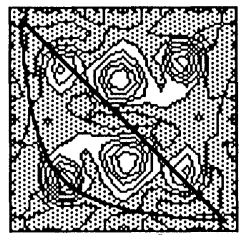

$\phi=86^{\circ}$
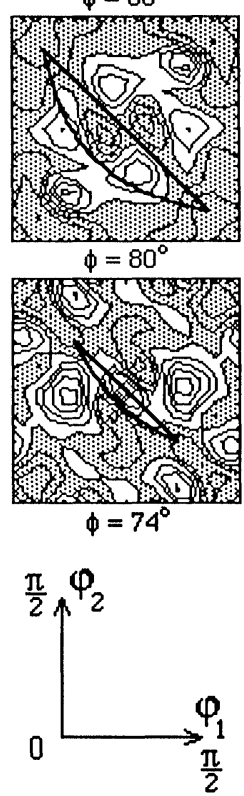

Figure 7 Rescaled Misorientation Correlation Function for first-neighbor misorientations associated with the $\mathrm{S}$ texture component. No misorientations less than $10^{\circ}$ are included. Contour levels of -1.5 , $-1.0,-0.5,0,0.5,1.0,1.5,2.0$ and 2.5 .

Both of these rotations are of interest in the investigation of the recrystallization process in deformed metals.

These MCFs show interesting features but were calculated from relatively few misorientation matrices. None of the five texture component MCFs mentioned were calculated from data sets with more than forty-five misorientation matrices whereas the MCF for the ODF peaks was obtained from 205 matrices. The low percentage of orientation pairs $\left(g_{A}, g_{B}\right)$ with $g_{A}$ in near coincidence with the five texture types requires further experiments to verify that these features are statistically reliable.

Aside from the appearance of the $60^{\circ}$ rotation about $\langle 111\rangle$ in several of the MCFs, the features of the misorientation textures associated with the $\mathrm{W}, \mathrm{C}, \mathrm{S}, \mathrm{B}$ and $G$ texture components exhibit several differences from those reported by Sztwiertnia and Haessner (1992). This is not necessarily surprising due to the twenty percent difference in strain level (i.e. $72.5 \%$ vs. $95 \%$ ). This difference in preferred misorientations at different strain levels should be of interest to investigators studying phenomena related to local misorientation, such as recrystallization. 
Table 2 Peak values from W, G and S MCF's in original Euler Angle and refined Axis-Angle parameterization.

\begin{tabular}{cl}
\hline Original peaks & Refined, axis-angle pairs \\
\hline Cube MCF & \\
$(25,72,60)$ & $40^{\circ}, \sim\langle 111\rangle$ \\
$(10,76,10)$ & $22^{\circ}, \sim\langle 111\rangle$ \\
$(15,78,75)$ & $22^{\circ}, \sim\langle 111\rangle$ \\
Goss MCF & \\
$(45,82,45)$ & $61^{\circ}, \sim\langle 332\rangle$ \\
$(40,76,40)$ & $60^{\circ}, \sim\langle 110\rangle$ \\
$(0,78,90)$ & $12^{\circ}, \sim\langle 100\rangle$ \\
$(50,72,25)$ & $46^{\circ}, \sim\langle 234\rangle$ \\
$\mathrm{S} \mathrm{MCF}$ & \\
$(20,90,70)$ & $30^{\circ}, \sim\langle 155\rangle$ \\
$(45,90,30)$ & $54^{\circ}, \sim\langle 123\rangle$ \\
$(20,86,70)$ & $30^{\circ}, \sim\langle 133\rangle$ \\
$(45,72,45)$ & $60^{\circ}, \sim\langle 111\rangle$ \\
$(65,72,5)$ & $29^{\circ}, \sim\langle 110\rangle$ \\
$(70,72,50)$ & $52^{\circ}, \sim\langle 124\rangle$ \\
\hline
\end{tabular}

\section{SUMMARY}

Use of the Textural Orientation Correlation reveals that in heavily deformed copper sheet there is a component in the misorientation texture which is not credited to the self-correlation of single grains and not predicted from the texture alone. This preferred misorientation corresponds to a spread around the $\Sigma 3$ misorientation in the general MCF for first-neighbors while more complicated features exist for misorientations associated with theoretical texture components. These observed relations between texture component type and preferred misorientation require further experimentation to determine their statistical verity as well as the possibility of further characterizing the incremental development of the misorientation textures.

\section{ACKNOWLEDGEMENT}

This work was sponsored by the National Science Foundation under a Materials Research Group Award (DMR-9001378).

\section{References}

Adams, B. L., Zhao, J. and Grimmer, H. (1990). Discussion of the representation of intercrystalline misorientation in cubic materials. Acta Crystographica A 46, 620-622.

Adams, B. L., Morris, P. R., Wang, T. T., Willden, K. S. and Wright, S. I. (1987). Description of Orientation Coherence in polycrystalline materials. Acta Metallurgica 35, 2935-2946.

Bunge, H.-J. (1982) Texture Analysis in Materials Science. Butterworths, London.

Haessner, F., Pospiech, J. and Sztwiertnia, K. (1983). Spatial Arrangement of Orientations in Rolled Copper. Materials Science and Engineering 57, 1-14. 
Hirsch, J. and Lücke, K. (1988). Overview No. 76 Mechanisms of Deformation and Development of Rolling Textures in Polycrystalline F.C.C. Metals-I. Description of Rolling Texture Development in Homogeneous CuZn Alloys. Acta Metallurgica 36, 2863-2882.

Plege, B. (1987). On orientation correlations and their relationship to texture. Theoretical Methods of Texture Analysis ed. H.-J. Bunge, DGM Informationsgesellschaft, Oberursel, 393-403.

Sztwiertnia, K. and Haessner, F. (1992). Orientation Characteristics of the Microstructure of Highly Rolled Pure Copper and Phosphorus Copper. Submitted to Textures and Microstructures.

Wang, T. T., Adams, B. L. and Morris, P. R. (1990). Development of Orientation Coherence in Plane-Strain Deformation. Metallurgical Transactions 21A, 2223-2236.

Zhao, J. and Adams, B. L. (1988). Definition of an Asymmetric Domain for Intercrystalline Misorientation in Cubic Materials in the Space of Euler Angles. Acta Crystallographica A 44, 326-336.

Zhao, J., Adams, B. L. and Morris, P. R. (1988). A Comparison of Measured and Texture-Estimated Misorientation Distributions in Type 304 Stainless Steel Tubing. Textures and Microstructures. 8-9, 493-508. 